\title{
Probability Evaluation Modeling and Planning of Product Disassembly Profit
}

\author{
Hongxing Deng ${ }^{1}$, Tiangang Qiang ${ }^{1}{ }^{*}$, Xiwang Guo ${ }^{2}$ and Ying Zhao ${ }^{1}$ \\ ${ }^{I}$ Transportation College, Northeast Forestry University, Harbin 150040, P. R. \\ China \\ ${ }^{2}$ Information Science Engineering College, Northeast University, Shenyang \\ 110819, P R. China \\ Email:1969dhx@163.com,qiangtg123@163.com,x.w.guo@163.com \\ zhaoying19901217@163.com
}

\begin{abstract}
Disassembly modeling and planning is meaningful and instructive for remanufacturing and recycling of disused products. However, an actual disassembly process of products has uncertainties since a variety of unpredictable factors. To handle such uncertainty, this work presents a novel disassembly modeling and planning approach using an extended stochastic Petri nets. An extended stochastic disassembly Petri nets (ESDPN) model is defined to provide basis for disassembly planning. Moreover, based on it, some typical probability evaluation models is built accordion to different disassembly criterion. In addition, a hybrid intelligent integrating stochastic simulation and neural network $(N N)$ is proposed to solve the proposed models, respectively. Some numerical examples are given to illustrate the proposed models and the effectiveness of proposed algorithms.
\end{abstract}

Keywords: Petri nets, discrete event system, disassembly, disassembly planning

\section{Introduction}

Due to growing concern and anxiety for material resources, energy conservation, and environmental protection, the recovery management of obsolete or disused products has attracted word-wide attention. Obviously, lots of obsolete products include a large number of renewable resources, e.g., gold, iron and aluminum, etc. additionally, some obsolete products may contain harmful and toxic matters, e.g., lead, chrome and obsolete products, etc., [1-3].

Thus, to establish a harmony life environment and promote the recycling and remanufacturing of obsolete products, it is natural that an efficient disassembly problem should be probed. One of important topics is disassembly modeling and planning. The early work mainly focused on determined problem. Disassembly modeling approach methods mainly include undirected graph, directed graph and AND/OR graph and Petri nets [2, 4-8]. Disassembly planning is considered as a NP problem and it aims to determine an optimal disassembly sequence. Many different solution methods have been formed and pretend in the field $[2,5]$. For example, some mathematical planning and recursive method, e.g, two-commodity network flow method [9] and rule-based recursive [10] approach. In addition, many intelligent algorithms have become a good tool to optimize the disassembly sequence, e.g., genetic algorithm [11], self-adaptive simplified swarm optimization method [12], improved max-min ant system [13] and heuristic methods [14].

However, disassembled products are usually ones used for a certain period of time, and thus can encounter much uncertainty. For instance, the condition of a returned product is uncertain since it is used for a long time and experiences some uncontrollable factors; the 
disassembly time of a product due to different disassembly tools and methods, and so on. since the emergence of these issues, some uncertainty management problems of product disassembly have been raised. Gungor and Gupta describe the uncertainty related difficulties in disassembly sequence planning. They present a methodology for disassembly sequence planning for products with defective parts in product recovery [15]. Ilgin and Gupta present a sensor embedded product approach to detect missing components on disassembly uncertainty [16-17]. They point out that a high degree of uncertainty exists in a disassembly system. The most popular approaches dealing with this uncertainty include simulation, stochastic programming, robust optimization, sensitivity and scenario analysis [18]. Tian et al. establish some tochastic programming models and perform the quantitative analysis of a disassembly process [19-21]. Sandborn and Murphy use Monte Carlo/ stochastic simulation to analyze the data uncertainty [22]. Kang et al. model disassembly operation times and recovery value in the form of intervals and apply the min-max regret criterion to get a robust disassembly sequence [23]. Tang and Zhou consider the uncertainty feature of disassembly time and quality of disassembled subassemblies in a disassembly process, and analyze the expected disassembly cost and expected net profit of product disassembly based on the generic model for a human-inthe-loop disassembly system [24]. They discuss one such system where a machine learning approach based on a disassembly Petri net (DPN) and a hybrid Bayesian network $(\mathrm{HBN})$ is used. In particular, this method models the disassembly process and predicts the outcome of each disassembly action by examining the probabilistic relationships between the different aspects of the disassembly process [25-26]. Reveliotis presents the uncertainty management in optimal disassembly planning through learning-based strategies [27]. Gao, et al., realize the intelligent decision making of a disassembly process based on fuzzy reasoning Petri nets [28].

Although some researchers have proposed to use Petri nets and fuzzy reasoning theory to address uncertainty management problems on disassembly, they pay little attention to the stochastic Petri nets modeling and planning issues of product disassembly. This work addresses modeling and planning methods for product disassembly based on extended stochastic Petri nets.

The rest of this paper is organized as follows. Section II states some assumptions and define a new disassembly model, i.e, extended stochastic disassembly Petri nets (ESDPN). Section III establishes probability Evaluation models of product disassembly profit based on ESDPN. Section IV introduces an algorithm to solve them. Section V presents some numerical examples to test its effectiveness. Finally, Section VI concludes this paper and describes future research issues.

\section{Problem Statement}

Disassembly is considered as a process to dismantling an assembly to its parts/components [29-30]. In this paper, we use a part or component or subassembly interchangeably as long as no confusion arises.

\subsection{Assumptions}

As the problem of disassembly is quite complex, for the purposes of this paper, several assumptions are taken into account during the formulation of disassembly planning problem: Disassembly of a product is carried out by a non-destructive disassembly approach; removal time is stochastic with known probability distribution; removal task failure rate and quality condition of parts are specified uniform distributions or interval numbers; and removal labor cost rate and part value are given.

\subsection{Extended Stochastic Disassembly Petri nets (ESDPN)}

The Petri net is a graphic modeling method, which is widely used in modeling and 
analyzing discrete event systems such as semiconductor manufacturing, transportation and automated manufacturing systems. An extended stochastic disassembly Petri nets (ESPN) is a high lever Petri net (PN), it is a type of improved stochastic Petri nets with arbitrary distribution. Basic PN knowledge can be referred to [31-32].

Based on the related concept of the ESPN, the extended stochastic disassembly Petri nets (ESDPN) of a product can be established according to the geometric constraint relationship, AND/OR logical relationship and removal state among various parts or components comprising a product.

Definition 1. An extended stochastic disassembly Petri nets (ESDPN) is defined as a ten-tuple

$$
\operatorname{ESDPN}=(S, T, I, O, M, x, \mathrm{c}, \tau, \delta, \rho)
$$

where

1) $S=\left\{s_{1}, s_{2}, \cdots, s_{n}\right\}$, is a finite set of places denoting the parts composing a product,

2) $T=\left\{t_{1}, t_{2}, \cdots, t_{s}\right\}$, is a finite set of transitions with $P \cup T=\Phi, P \cap T=\Phi$, it denotes the removal operation of the part.

3) $I: S \times T \rightarrow N$, is an input function that defines the set of directed arcs from $\mathrm{P}$ to $\mathrm{T}$,

4) $O: T \times S \rightarrow N$, is an output function that defines the set of directed arcs from $\mathrm{T}$ to $\mathrm{S}$. Note that that $A \subseteq\{S \times T\} \cup\{T \times S\}$ is a set of directed arcs denoting the priority removal relation of various part composing a product,

5) $M: S \rightarrow N$, Mi is a marking represents the number of tokens in the ith place. An initial marking is denoted by M0,

6) $x: T \rightarrow \xi$ is a removal time probability density function associated with a transition, where $\xi$ is the set of random variable denoting the needed time to removal a specified part,

7) $c: T \rightarrow R^{+}$is a removal cost rate associated with a transition, where $\mathrm{R}+$ is the set of non-negative real numbers,

8) $\tau: T \rightarrow R^{+}$is a removal value associated with a transition, where $R^{+}$is the set of nonnegative real numbers,

9) $\delta: T \rightarrow \xi$ is quality condition function associated with a transition, where $\xi \sim U[0,1]$ and it is the set of random uniform variable. The vale ${ }^{\xi(t)}$ denotes the quality condition of a removed part. Clearly, $\xi(t)=0$ means that the quality condition of a part is worst, i.e., it has no value; while $\xi(t)=1$ means that the quality condition of a part is best, i.e., it is intact, and,

10) $\rho: T \rightarrow[0.85,1]$ is a probability value associated with a transition. It is updated according to the sensing result of the corresponding disassembly operation performed by external resources. The value ${ }^{\rho(t)}$ represents the success rate of a removal operation. The value of $1-\rho(t)$ represents the failure rate. ${ }^{\rho(t)}$ is assumed to be $\rho(t) \in[0.85,1]$ since most of removal part can be successfully obtained in reality in this paper.

Based on the ESDPN and reachability of PN, a feasible disassembly sequence is presented next,

An ESPN of a product is shown in Fig. 1. We can see that it clearly describe the removal relation of various parts comprising the product using its related elements. The set of places $S=\left\{\mathrm{s}_{1}, \mathrm{~s}_{2}, \mathrm{~s}_{3}, \mathrm{~s}_{4}, \mathrm{~s}_{5}, \mathrm{~s}_{6}, \mathrm{~S}_{7}\right\}$ denote seven parts comprising this product, i.e., parts 1 , $2,3,4,5,6$, and 7 . The set of transitions $T=\left\{t_{1}, t_{2}, t_{3}, t_{4}, t_{5}, t_{6}, t_{7}\right\}$ denotes the removal operation of corresponding part. For example, $t_{1}$ denotes removing part 1 denoted by $s_{1} ; t_{2}$ denotes removing parts 2 or 3 due to it is a shared transition. Arc set $A=\left\{\left(\mathrm{s}_{1}, \mathrm{t}_{1}\right),\left(\mathrm{t}_{1}, \mathrm{~s}_{2}\right)\right.$, $\left(\mathrm{s}_{2}, \mathrm{t}_{2}\right),\left(\mathrm{t}_{1}, \mathrm{~s}_{3}\right),\left(\mathrm{s}_{3}, \mathrm{t}_{2}\right),\left(\mathrm{t}_{2}, \mathrm{~s}_{5}\right),\left(\mathrm{s}_{5}, \mathrm{t}_{4}\right),\left(\mathrm{t}_{4}, \mathrm{~s}_{6}\right),\left(\mathrm{t}_{1}, \mathrm{~s}_{4}\right),\left(\mathrm{s}_{4}, \mathrm{t}_{3}\right),\left(\mathrm{t}_{3}, \mathrm{~s}_{6}\right),\left(\mathrm{s}_{6}, \mathrm{t}_{5}\right),\left(\mathrm{t}_{5}, \mathrm{~s}_{7}\right),\left(\mathrm{s}_{7}, \mathrm{t}_{6}\right),\left(\mathrm{t}_{6}, \mathrm{~s}_{8}\right)$, $\left.\left(\mathrm{s}_{8}, \mathrm{t}_{7}\right),\left(\mathrm{t}_{7}, \mathrm{~s}_{1}\right)\right\}$ denotes the priority removal relation of various part composing this product. 
For example, the arc $\left\{\left(\mathrm{s}_{1}, \mathrm{t}_{1}\right),\left(\mathrm{t}_{1}, \mathrm{~s}_{2}\right)\right\}$ denotes part 1 should be removed before part 2 , denoted by $s_{2}$. In addition, two subset of the ESPN, i.e., its "AND" and "OR" structures are presented in Figure 2. In "AND" structure shown in Figure 2(a), $\operatorname{arcs}\left\{\left(\mathrm{s}_{2}, \mathrm{t}_{2}\right),\left(\mathrm{t}_{2}, \mathrm{~s}_{5}\right)\right\}$ and $\left\{\left(\mathrm{s}_{3}, \mathrm{t}_{2}\right),\left(\mathrm{t}_{2}, \mathrm{~s}_{5}\right)\right\}$ make up a logical "AND" relationship, which denotes part 5 can be removed after parts 2 and 3 are removed in a disassembly process. In "OR" structure shown in Figure 2(b), arcs $\left\{\left(\mathrm{s}_{5}, \mathrm{t}_{4}\right),\left(\mathrm{t}_{4}, \mathrm{~s}_{6}\right)\right\}$ and $\left\{\left(\mathrm{s}_{4}, \mathrm{t}_{3}\right),\left(\mathrm{t}_{3}, \mathrm{~s}_{6}\right)\right\}$ make up a logical "OR" relationship, which denotes part 6 can be removed after either part is removed [33-34]. Note that the place with initial marking M0 is the removal source place.

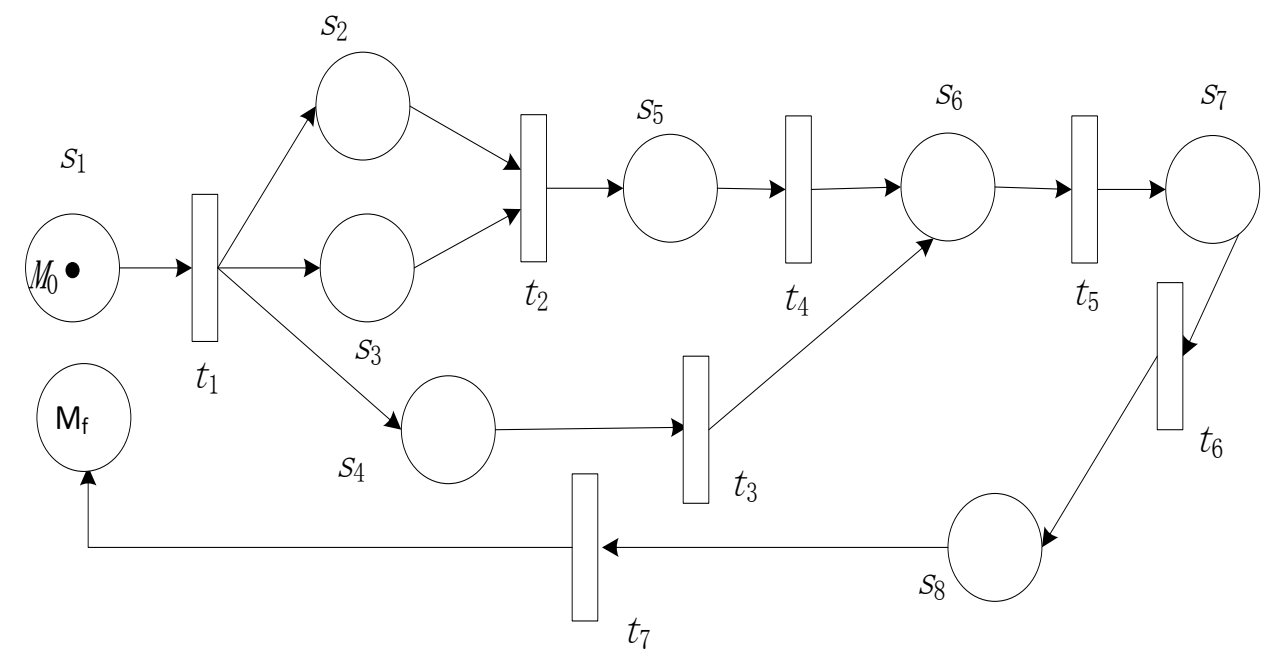

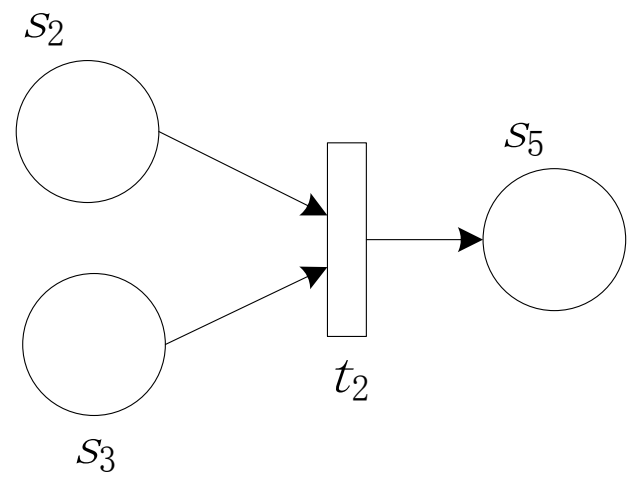

(a) "AND" structure

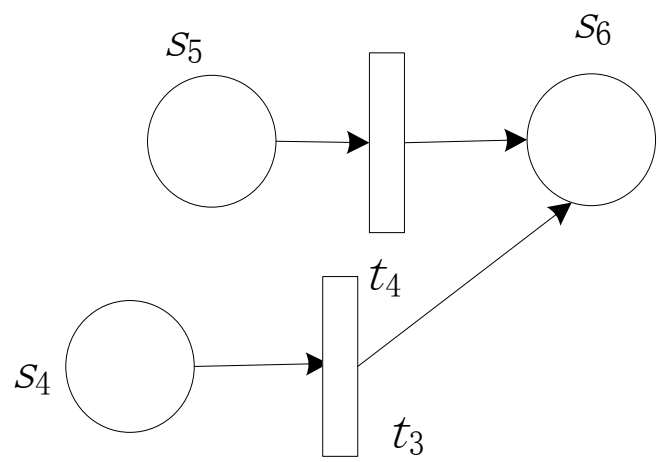

(b) "OR" structure

Figure 2. Schematic diagram of "AND" and "OR" of an ESPN

Let $\sigma$ be Parikh vector. In addition, Let a disassembly decision-maker want to obtain part 7, based on Definition 2, there are two feasible paths, i.e.,

Path I: $1 \rightarrow 4 \rightarrow 6 \rightarrow 7$;

Path II: $1 \rightarrow\{2,3\} \rightarrow 5 \rightarrow 6 \rightarrow 7$.

Their responding firing transition sequence $\sigma_{1}(t)$ and $\sigma_{2}(t)$ are expressed as, $\sigma_{1}(t)=\mathrm{t}_{1} \mathrm{t}_{3} \mathrm{t}_{5} \mathrm{t}_{6} ;$ $\sigma_{2}(t)=\mathrm{t}_{1} \mathrm{t}_{2} \mathrm{t}_{4} \mathrm{t}_{5} \mathrm{t}_{6} ;$

Thus their Parikh vectors $\vec{\sigma}_{1}(t)$ and $\vec{\sigma}_{2}(t)$ are presented next, respectively, $\vec{\sigma}_{1}(t)=\left[\begin{array}{lllllll}1 & 0 & 1 & 0 & 1 & 1 & 0\end{array}\right] \mathrm{T}$;

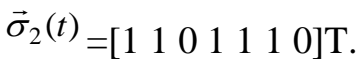


Moreover, based on the Definition 1., the removal cost $\mathrm{C}_{\mathrm{i}}\left(\mathrm{t}_{\mathrm{i}}\right)$ and actual recovery value $\mathrm{R}_{\mathrm{i}}\left(\mathrm{t}_{\mathrm{i}}\right)$ of ith part associated with ith transition in a feasible path are expressed as, respectively,

$$
\begin{gathered}
C_{i}\left(t_{i}\right)=c_{i} x_{i} \\
R_{i}\left(t_{i}\right)=\rho_{i} \delta_{i} \tau_{i}
\end{gathered}
$$

where $\mathrm{x}_{\mathrm{i}}, \mathrm{c}_{\mathrm{i}}, \rho_{i}, \delta_{i}$, and $\pi_{i}$ are the removal time, labor cost rate, removal success rate, and removal value to remove ith part.

The needed disassembly time $\mathrm{X}(\mathrm{t})$ to remove a specified part along a disassembly sequence fired by transition sequence $\sigma$ is expressed as,

$$
X(t)=\sum_{i=1}^{k} x_{i}=X \times \vec{\sigma}
$$

where $\mathrm{k}$ is the number of parts in a feasible disassembly path; and $X=\left\{x_{1}, x_{2}, \cdots, x_{i}, \cdots x_{k}\right\}$ is a matrix of removal time. As shown in Fig. 1., for path I to obtain part $7, X_{1}=\left[x_{1}, x_{2}, 0, x_{4}, x_{5}, x_{6}, 0\right]$, thus, based on Equation (6), the needed disassembly time to remove part $7 \mathrm{X}_{1}(\mathrm{t})=\mathrm{X}_{1} \times \vec{\sigma}_{1}=\left[\begin{array}{lllllll}\mathrm{x}_{1} & \mathrm{x}_{2} & \mathrm{x}_{3} & \mathrm{x}_{4} & \mathrm{x}_{5} & \mathrm{x}_{6} & \mathrm{x}_{7}\end{array}\right] \times\left[\begin{array}{llllll}1 & 0 & 1 & 0 & 1 & 1\end{array}\right.$ $0] \mathrm{T}=\mathrm{x}_{1}+\mathrm{x}_{3}+\mathrm{x}_{5}+\mathrm{x}_{6}$.

Similarly, the needed disassembly cost $\mathrm{C}(\mathrm{t})$, recovery value $\mathrm{R}(\mathrm{t})$, and net profit $\mathrm{B}(\mathrm{t})$ to remove a specified part along a disassembly sequence fired by transition sequence $\sigma$ are expressed next, respectively,

$$
\begin{aligned}
& C(t)=C \times \vec{\sigma} \\
& R(t)=R \times \vec{\sigma}
\end{aligned}
$$

where $\mathrm{C}$ and $\mathrm{R}$ are matrixes of the removal cost and recovery value. Note that, if there is a "AND" structure/ shared transition in a feasible disassembly path, the removal cost and recovery value of responding transition should be superposed when obtaining $\mathrm{C}(\mathrm{t})$ and $\mathrm{R}(\mathrm{t})$.

Thus, the net benefit/profit $\mathrm{B}(\mathrm{t})$ to remove a specified part along a disassembly sequence fired by transition sequence $\sigma$ is expressed as,

$$
B(t)=C(t)-R(t)=R \times \vec{\sigma}-C \times \vec{\sigma}=(C-R) \times \vec{\sigma}
$$

\subsection{Basic Concepts}

Disassembly depth refers to the extent to which a product is disassembled. This work proposes the concept of disassembly ratio to measure it.

Definition 3. Disassembly ratio is defined as the ratio between the number of parts that can be disassembled by a non-destructive disassembly method and the total number of parts comprising a whole product. Mathematically,

$$
\lambda=\left(N_{d} / N_{0}\right) \times 100 \%
$$

where $N_{d}$ is the number of parts that can be disassembled, and $N_{0}$ is the total number of parts comprising a whole product. Clearly, $\lambda=1$ denotes that a product can be disassembled completely. $\lambda=0$ means that a product cannot be disassembled. Disassembly ratio denotes a pre-given target of a disassembly task by a ratio.

Definition 4. Disassemblability degree is defined as the probability for a product to reach a disassembly ratio given a specified time constraint.

Let random variable ${ }^{X_{\lambda}}$ denote the needed disassembly time for reaching a specified disassembly ratio $\lambda$. Then the product disassemblability degree at given time $\mathrm{x}$ is the probability of random variable $X_{\lambda} \leq x$, namely,

$$
D_{\lambda}(x)=P\left(X_{\lambda} \leq x\right)
$$


If $D_{\lambda}(x)$ is continuous and differentiable, then the disassembly probability density function $f_{d}(x)$ is expressed as

$$
f_{d}(x)=d D_{\lambda}(x) / d x
$$

Namely,

$$
D_{\lambda}(x)=\int_{0}^{x} f_{d}(\xi) d \xi
$$

Obviously, $D_{\lambda}(x)=1$ denotes that a product certainly reaches a specified disassembly ratio while $D_{\lambda}(x)=0$ means that a product cannot be disassembled to reach such ratio.

Definition 5. Cost disassemblability degree is defined as the probability for a product to reach a disassembly depth given a specified cost constraint.

Let random variable $C_{\lambda}$ denote the needed disassembly cost to reach a specified disassembly ratio $\lambda$. The product's cost disassemblability degree at cost $\mathrm{c}$ is the probability of random variable $C_{\lambda} \leq c$,

$$
G_{\lambda}(c)=P\left(C_{\lambda} \leq c\right)
$$

If $G_{\lambda}(c)$ is continuous and differentiable, its density function $f_{g}(c)$ is expressed as

$$
f_{g}(c)=d G_{\lambda}(c) / d c
$$

That is,

$$
G_{\lambda}(c)=\int_{0}^{c} f_{g}(\xi) d \xi
$$

Definition 6. Profit disassemblability degree is defined as the probability for a product to reach a disassembly depth obtained a specified profit constraint.

Let random variable ${ }^{B_{\lambda}}$ denote the obtained net profit to reach a specified disassembly ratio $\lambda$. The product's profit disassemblability degree at profit $b$ is the probability of random variable $B_{\lambda} \geq b$,

$$
U_{\lambda}(c)=P\left(B_{\lambda} \geq b\right)
$$

If $U_{\lambda}(b)$ is continuous and differentiable, its density function $f_{u}(b)$ is expressed as

$$
f_{u}(b)=d G_{\lambda}(b) / d b
$$

That is,

$$
U_{\lambda}(b)=\int_{0}^{b} f_{u}(\xi) d \xi
$$

By taking the single target profit disassemblability degree as an example, this paper presents its profit evaluation models and analysis results next.

\section{Probability Evaluation Models of Product Disassembly Profit based on ESDPN}

According to defined ESDPN and disassembly evaluation parameters, four types of single-objective stochastic models for profit disassemblability degree analysis of product disassembly can be built, namely, i.e., the general maximum profit disassemblability degree model, the maximum profit disassemblability degree model with a disassembly time constraint, the maximum profit disassemblability degree model with a disassembly cost constraint, and the maximum profit disassemblability degree model with disassembly time and cost constraints.

In ESDPN, a disassembly decision-maker hopes to seek the maximum probability of removing a target part in the obtained disassembly profit under limited resources. In order to determine the optimal disassembly sequence, it is necessary to build the general maximum profit disassemblability degree model for disassembly decision-making. The objective function of this model is written as: 
Subject to:

$$
\max \left(U_{\lambda}^{b}\right)
$$

$$
M^{\prime}=M_{0}+D \times \vec{\sigma}
$$

\section{Solution Method}

In order to solve our proposed models, a hybrid intelligent algorithm integrating stochastic simulation and neural networks $(\mathrm{NN})$ is introduced in this paper, i.e., $\mathrm{NN}$ based simulation algorithm, in which stochastic simulation is used to estimate the related uncertain functions [35-37] and $\mathrm{NN}$ is used to fit their nonlinear fitting ability [38-42].

In above established models, there are three stochastic functions, namely, the probability of disassembly time of a disassembly process, i.e., disassemblability degree $D_{\lambda}$; the probability of disassembly cost of a disassembly process, i.e., cost disassemblability degree $G_{\lambda}$; and the probability of obtaining disassembly profit of a disassembly process, i.e., $H_{\lambda}$. The calculation of these uncertain functions can be easily achieved by stochastic simulation. Please refer to our prior works in detail [21, 43]. NN is treated as a nonlinear mapping system composed of neurons, which are linked by weighted connections. It is usually compsoed of three layers: input, hidden, and output layers. It has strong nonlinear fitting ability, its basic knowledge can be referred to [3940], [43-45].

Steps of NN-based stochastic evaluation algorithm are presented as follows:

Step 1: Establish an ESDPN according to the topology and constraint relationships between parts of a product.

Step 2: Initialize parameters of the $\mathrm{NN}$ structure, the number of training data $K$, prameters $x_{i}, c_{i} \rho_{i}, \delta_{i}$ and $\tau_{i}$ associated with each transition in ESDPN, and set a target part j.

Step 3: Based on an ESDPN and generate various firing transition sequence of target part, i.e, its various feasible disassembly path is obtained.

Step 4: Based on the relationship among removal time, removal cost, removal value and disassembly net profit of a feasible disassembly path, generate the input-output data of NN training by stochastic simulation technology. If there are constraints, e.g., $P\left\{X_{\lambda}(t) \leq X^{0}\right\} \geq D_{\lambda}$ or $P\left\{C_{\lambda}(t) \leq C^{0}\right\} \geq G_{\lambda}$ the constraints need to be checked by stochastic simulation.

Step 5: Train a neural network to approximate the uncertain function, namely net profit function and obtain output data of disassembly net profit of the neural network.

Step 6: Calculate the profit disassemblability degree, or profit disassemblability degree model with disassembly time or cost constraints, or profit disassemblability degree model with disassembly time and cost constraints of each feasible disassembly sequence, and seek their maximum value, namely $\max \left(U_{\lambda}^{b}\right)$ is obtained.

The above algorithm has been implemented in the MATLAB (R2009b) programming language.

\section{Case Study}

Consider a disassembly problem of a oil fan clutch in a vehicle as shown in Figure 3. According to the geometric constraint relationship, AND/OR logical relationship and removal state among various parts of a silicone oil fan clutch, its ESDPN is established and shown in Figure 4. Note that the number of bolts I and II is 6 and 8 in this clutch, respectively. 


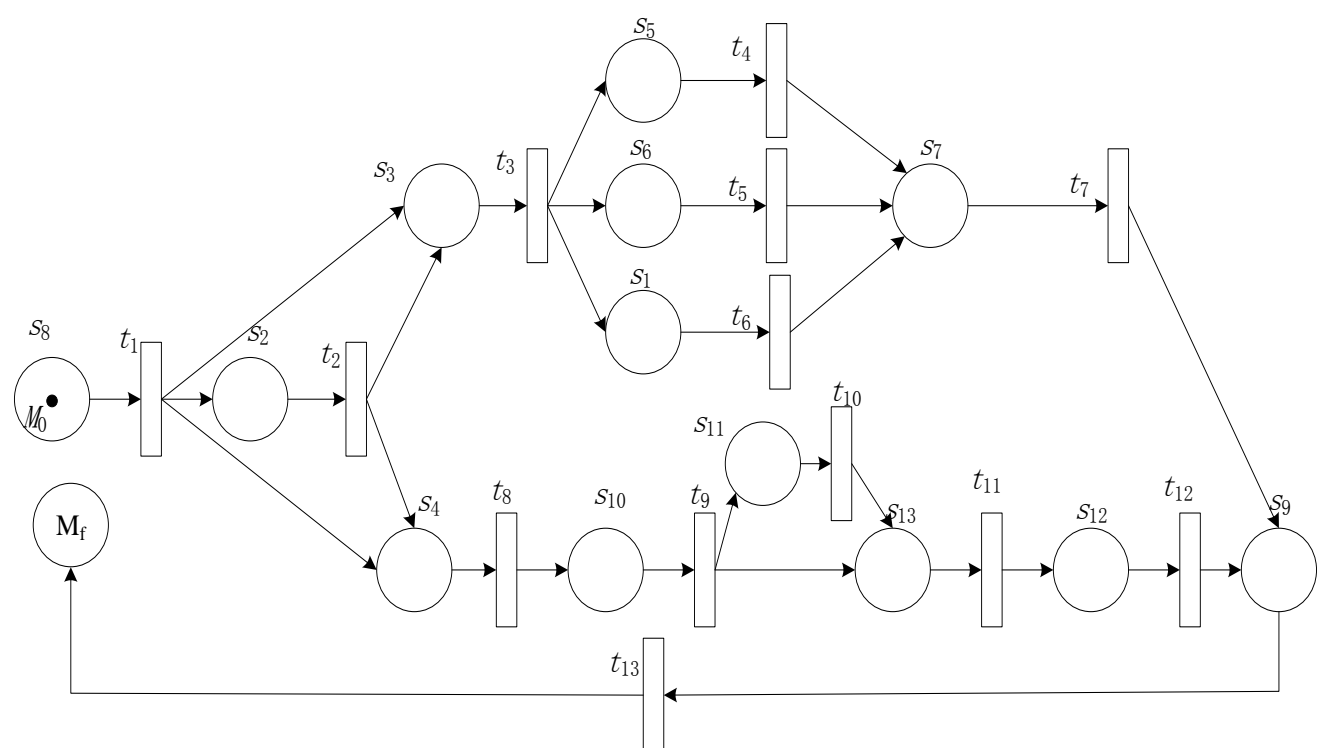

Figure 3. ESDPN Model of an Oil Fan Clutch

In addition, the paraphrase for the transitions and places of ESDPN model of the silicone oil fan clutch is listed Tables I and II, respectively. Additionally, the parameters associated with each transition is listed Table II. Note that the unit of time is Second (s), the unit of labor cost is Yuan/Second, disassembly cost and net profit are Yuan. $U(a, b)$ and $\operatorname{Logn}(\mu, \sigma)$ denote uniform and lognormal distributions, respectively. These distribution types result from the assumption of related work [2, 21, 46]-[50]. In addition, the quality condition function and the success rate of a removal operation associated with a transition is assumed to be $\delta \sim U[0,1]$ and $\rho \sim U[0.85,1]$ when executing the removal operation.

Table 1. The Paraphrase for the Transitions and Places of ESDPN Model of Silicone Oil Fan Clutch

\begin{tabular}{cc}
\hline Place & Paraphrase \\
\hline$s_{1}$ & Part 1, i.e., check valve \\
$s_{2}$ & Part 2, i.e., driven disc \\
$s_{3}$ & Part 3, i.e., control valve flake \\
$s_{4}$ & Part 4, i.e., bolt I \\
$s_{5}$ & Part 5, i.e., valve drive pin \\
$s_{6}$ & Part 6, i.e., bimetal temperature sensor \\
$s_{7}$ & Part 7, i.e., front cover \\
$s_{8}$ & Part 8, i.e., bolt II \\
$s_{9}$ & Part 9, i.e., clutch housing \\
$s_{10}$ & Part 10, i.e., active board \\
$s_{11}$ & Part 11, i.e., ring \\
$s_{12}$ & Part 12, i.e., bearing \\
$s_{13}$ & Part 13, i.e., drive shaft \\
\hline
\end{tabular}


Table 2. Paraphrases of Each Transition and its Related Parameters ESPN Models of Silicone Oil Fan Clutch

\begin{tabular}{|c|c|c|c|c|}
\hline Transition & Paraphrase & $\begin{array}{l}\text { Removal time } \\
\text { function } f(x)\end{array}$ & $\begin{array}{l}\text { Removal } \\
\text { cost rate } c\end{array}$ & Value $\delta$ \\
\hline$t_{1}$ & Removal part 8 & $\log n(\log (12), 0.2)$ & 0.12 & 2.5 \\
\hline$t_{2}$ & Removal part 2 & $U(6,8)$ & 0.1 & 20 \\
\hline$t_{3}$ & Removal part 3 & $U(5,7)$ & 0.1 & 9 \\
\hline$t_{4}$ & Removal part 5 & $U(4,6)$ & 0.1 & 10 \\
\hline$t_{5}$ & Removal part 6 & $U(3,5)$ & 0.1 & 15 \\
\hline$t_{6}$ & Removal part 1 & $U(2,4)$ & 0.1 & 10 \\
\hline$t_{7}$ & Removal part 7 & $U(7,9)$ & 0.1 & 20 \\
\hline$t_{8}$ & Removal part 4 & $\log n(\log (10), 0.2)$ & 0.11 & 2.0 \\
\hline$t_{9}$ & Removal part 10 & $U(11,13)$ & 0.1 & 11 \\
\hline$t_{10}$ & Removal part 11 & $U(2,4)$ & 0.1 & 1.5 \\
\hline$t_{11}$ & Removal part 13 & $U(10,15)$ & 0.1 & 13 \\
\hline$t_{12}$ & Removal part 12 & $\log n(\log (18), 0.2)$ & 0.13 & 9 \\
\hline$t_{13}$ & Removal part 9 & $u(9,13)$ & 0.1 & 25 \\
\hline
\end{tabular}

According to ESDPN of a silicone oil fan clutch in a vehicle, i.e., Fig. 4., there are six feasible paths to remove part 7 when it is disassembled, namely,

Path I $8 \rightarrow 3 \rightarrow 5 \rightarrow 7$;

Path II $8 \rightarrow 3 \rightarrow 6 \rightarrow 7$;

Path III $8 \rightarrow 3 \rightarrow 1 \rightarrow 7$;

Path V $8 \rightarrow 2 \rightarrow 3 \rightarrow 5 \rightarrow 7$;

Path VI $8 \rightarrow 2 \rightarrow 3 \rightarrow 6 \rightarrow 7$

Path VII $8 \rightarrow 2 \rightarrow 3 \rightarrow 1 \rightarrow 7$.

Their responding firing transition sequence are expressed as,

$\sigma_{1}(t)=\mathrm{t}_{1} \mathrm{t}_{3} \mathrm{t}_{4} \mathrm{t}_{7} ;$

$\sigma_{2}(t)=\mathrm{t}_{1} \mathrm{t}_{3} \mathrm{t}_{5} \mathrm{t}_{7}$;

$\sigma_{3}(t)=\mathrm{t}_{1} \mathrm{t}_{3} \mathrm{t}_{6} \mathrm{t}_{7}$;

$\sigma_{4}(t)=\mathrm{t}_{1} \mathrm{t}_{2} \mathrm{t}_{3} \mathrm{t}_{4} \mathrm{t}_{7} ;$

$\sigma_{5}(t)=\mathrm{t}_{1} \mathrm{t}_{2} \mathrm{t}_{3} \mathrm{t}_{5} \mathrm{t}_{7}$

$\sigma_{6}(t)=\mathrm{t}_{1} \mathrm{t}_{2} \mathrm{t}_{3} \mathrm{t}_{6} \mathrm{t}_{7}$.

The parameters of the NN based simulation algorithm are set next in this paper: for the above identified Paths I, II and III, set the number of input neurons, i.e., the number of firing transition in a specified disassembly path $u=4$, while the others are 5 , and the number of output neurons $v=1$, which representing one disassembly net profit function. Thus the number of hidden neurons is 12 by letting $b=12$. The given error term value is 0.000004. Based on the stochastic simulation, 4000 input-output data points are generated and obtained. Moreover, they are separated into two groups: 3000 data points for training and 1000 for testing. Note that the number of training data points is set to be 3000 due to two reasons. For one thing, too few data points make the solution of models lack accuracy. For another thing, too many increase the training time and response time of the solution model, moreover, note that the solution accuracy cannot be increased significantly with the increase of the number of data [37].

Example 1. A disassembly decision-maker hopes to get the maximum profit disassemblability degree to remove a target part 7 obtained 10 Yuan profit constraint. Set the corresponding parameters of the proposed model presented in Section III.A and use the proposed algorithm. The optimal solution of this problem can be obtained, namely, the optimal firing transition sequence is $\sigma_{5}(t)=\mathrm{t}_{1} \mathrm{t}_{2} \mathrm{t}_{3} \mathrm{t}_{5} \mathrm{t}_{7}$, i.e., the optimal disassembly path 
Path VI: $8 \rightarrow 2 \rightarrow 3 \rightarrow 6 \rightarrow 7$, and the maximum profit disassemblability degree is 0.914 . Put differently, the maximum probability to remove a target part 7 obtained 10 Yuan net profit is 0.914 along this best disassembly sequence.

In addition, in order to test the effectiveness of algorithms and correctness of the results, the calculation results of running 6 times of Example 1 of the used NN-based stochastic evaluation algorithm is shown in Table III. Their relative error is obtained shown in Table III and it is defined as ((Optimal result-Actual result)/Optimal result $\times 100 \%$ ), where optimal result is the maximum value of solution results of the model with different run times while actual results are the solution results of the model when the algorithm is run each time.

Table 3. Comparison of Solutions of Example I

\begin{tabular}{cccc}
\hline Times & Actual result & Optimal result & Relative error $(\%)$ \\
\hline 1 & 0.9140 & 0.9250 & 1.10 \\
2 & 0.9210 & 0.9250 & 0.40 \\
3 & 0.9200 & 0.9250 & 0.50 \\
4 & 0.9050 & 0.9250 & 2.00 \\
5 & 0.9020 & 0.9250 & 2.30 \\
6 & 0.9250 & 0.9250 & 0.00 \\
\hline
\end{tabular}

From Table III, the maximum relative error does not exceed $2.5 \%$. It shows that the used algorithm is feasible when used to proposed model.

Additionally, to observe the effectiveness of the proposed methods, its predicted outputs and their output error between forecast and actual outputs of test data for the 1th running result on Example $\mathbf{I}$ are shown in Figures. 4 to 5.

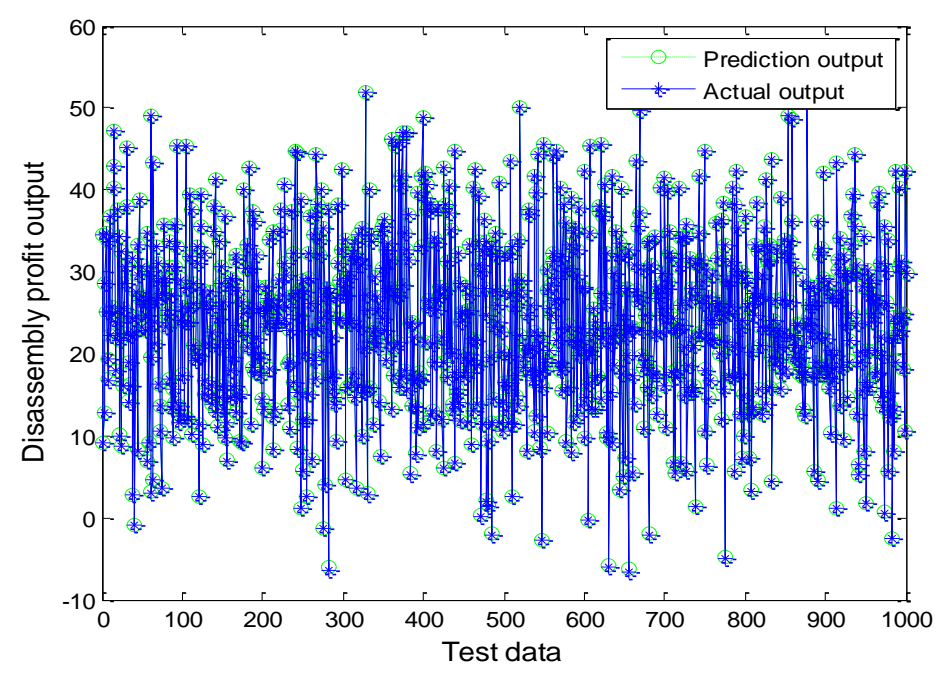

Figure 4. Prediction Output of NN-based Stochastic Evaluation Algorithm 


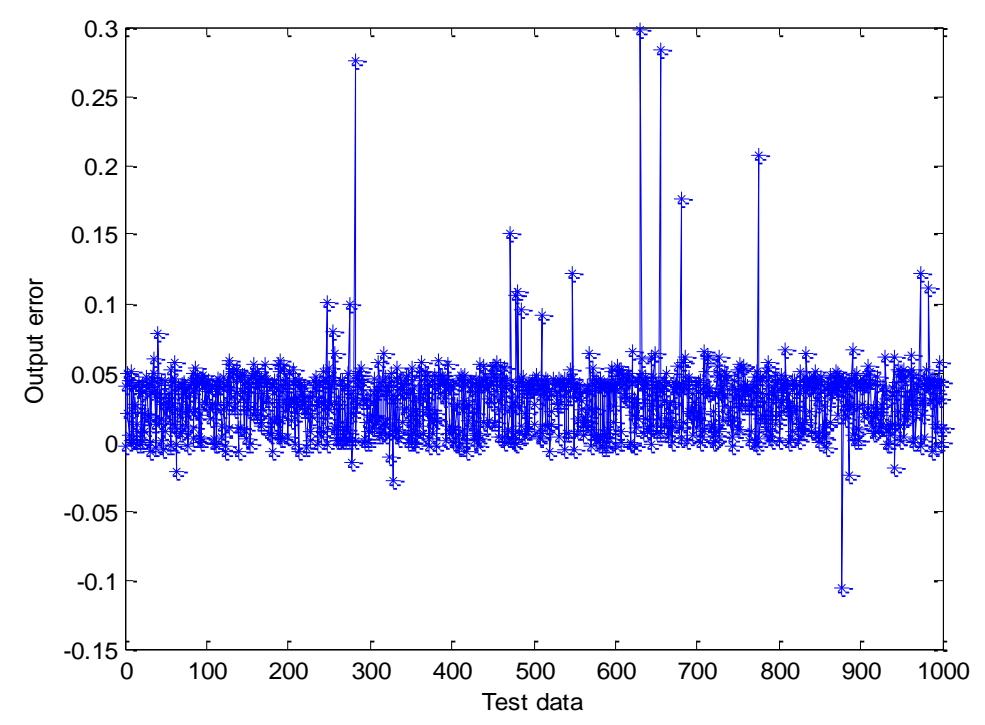

Figure 5. Error Output of NN-based Stochastic Evaluation Algorithm

From Figures. 4 to 5, it can be seen that the prediction output and actual output are close, moreover, the absolute of their error does not exceed 0.3. It reveals that the algorithm is highly satisfactory when it is used to solve the profit disassemblability degree models.

\section{Conclusions}

Disassembly modeling and optimization is one of important topics in the sustainable/green manufacturing field. However, obsolete products often suffer from a variety of uncertainties. To handle it, this work proposes a novel extended stochastic disassembly Petri nets (ESPN) approach to model a disassembly process for the first time. According to defined ESPN and different disassembly constraints, four models for profit disassemblability degree analysis of product disassembly are built, namely, i.e., the general maximum profit disassemblability degree model. In addition, stochastic simulation algorithms based on NN are adopted to solve the proposed models. The results reveal that they are feasible when used to solve the proposed models.

The future work is to find and use actual disassembly data to validate this method to provide the best decision support for disassembly practice. In addition, the evaluation and optimization of large-scale obsolete products should be further discussed.

\section{References}

[1] Y. Tang and M . C. Zhou, "Learning-Embedded Disassembly Petri Net for Process Planning," IEEE Int. Conf. on Syst., Man, and Cyber., Taipei, Taiwan, (2006) October, pp. 80-85.

[2] Y. Tang, M. C. Zhou, E. Zussman, and R. J. Caudill, "Disassemblymodeling, planning, and application: A review", in Proc. IEEE Int. Conf. Robot. Autom., San Francisco, CA, USA, (2000) April, pp. 21973002.

[3] G. D. Tian, Y. M. Liu, H. Ke and J. W. Chu, "Energy evaluation method and its optimization models for process planning with stochastic characteristics: A case study in disassembly decision-making," Comput. Ind. Eng., vol. 63, (2012), pp. 553-563.

[4] A. Gungor and S. M. Gupta, "An evaluation methodolgy for disassembly prccesses," Comput. Ind. Eng., vol. 33, no. 1-2, (1997), pp. 329-332.

[5] H. C. Zhang, T. C. Kuo, H. Lu and S. H. Huang, "Environmentally conscious design and manufacturing: a state-of-the-art survey,” J. Manuf. Syst., vol. 16, (1997), pp. 352-371.

[6] E. Zussman and M. C. Zhou, "A Methodology for Modeling and Adaptive Planning of Disassembly Processes”, IEEE Trans. Robot. Autom., vol, 15, no. 1, (1999) Febraury, pp. 190-194. 
[7] Y. Tang, M. C. Zhou and R. J. Caudill, "An Integrated Approach to Disassembly Planning and Demanufacturing Operation," IEEE Trans. Robot. Autom., vol, 17, no. 6, (2001) December, pp. 773784.

[8] M. Gao, M. C. Zhou and R. J. Caudill, "Integration of Disassembly Leveling and Bin Assignment for Demanufacturing Automation,” IEEE Trans Robot Autom., vol. 18, no.6, pp. 867-874, Dec. 2002.

[9] A. J. D. Lambert, “Optimal disassembly of complex products,” Int. J. Prod. Res., vol. 35, pp. 2509-2523, 1997.

[10] S. S. Smith and W. H. Chen, "Rule-based recursive selective disassembly sequence planning for green design,” Adv. Eng. Inform., vol. 25, pp. 77-87, 2011.

[11] J. R. Li, L. P. Khoo and S. B. Tor, "An object-oriented intelligent disassembly sequence planner for maintenance," Comput. Ind., vol. 56, pp. 699-718, 2005.

[12] X. H. Liu, G. L. Peng, X. M. Liu and Y. F. Hou, "Disassembly sequence planning approach for product virtual maintenance based on improved max-min ant system," Int. J. Adv. Manuf. Technol., vol. 59, pp. 829-839, 2012

[13] W. C. Yeh, "Optimization of the disassembly sequencing problem on the basis of self-adaptive simplified swarm optimization,” IEEE Trans. Syst. Man, Cybern. Part A: Syst. Humans, vol. 42, no. 1, pp. 250-261, Jan. 2012.

[14] B. Adenso-Diaz, S. Garcia-Carbajal, and S. M. Gupta, "A path-relinking approach for a bi-criteria disassembly sequencing problem,” Comput. Oper. Res., vol. 35, pp. 3989-3997, 2008.

[15] A. Gungor and S. M. Gupta, "Disassembly sequence planning for products with defective parts in product recovery," Comput. Ind. Eng., vol. 35, pp. 161-164, 1998.

[16] M. A. Ilgin and S. M. Gupta, "Performance Improvement Potential of Sensor Embedded Products in Environmental Supply Chains”, Resour., Conserv. Recy., vol 55, pp. 580-592, 2011.

[17] M. A. Ilgin and S. M. Gupta, "Recovery of Sensor Embedded Washing Machines Using a Multi Kanban Controlled Disassembly Line", Rot. Cim-Int. Mauf., vol 27, pp. 318-334, 2011.

[18] M. A. Ilgin and S. M. Gupta, "Environmentally conscious manufacturing and product recovery (ECMPRO): A review of the state of the art," J. Environ. Manage., vol 91, pp. 563-591, 2010

[19] G. D. Tian, Y. M. Liu, Q. Z. Tian and J. W. Chu, "Evaluation model and algorithm of product disassembly process with stochastic feature," Clean Techn. Environ. Policy, vol. 14, pp. 345-356, 2012.

[20] R. J. Liu, G. D. Tian, X. Y. Zhang, A. Y. Zhao, X. L. Wang and Q. N. Niu, "Disassembly Sequence Optimization for Automotive Product Based on Probabilistic Planning Method," in Proc. Int. Conf. Consum. Electr.,Commun. Netw. (CECNet 2011), Xianning, China, vol. 1, pp. 284-288, Apr. 2011.

[21] G. D. Tian, M. C. Zhou, J. W. Chu and Y. M. Liu, "Probability evaluation models of product disassembly cost subject to random removal time and different removal labor cost," IEEE Trans. Autom. Sci. Eng., vol. 9, no. 2, pp. 288-295, Apr. 2012.

[22] P. A. Sandborn and C. F. Murphy, "A model for optimising the assembly and disassembly of electronic systems," IEEE Trans. Electr. Pack. Manuf., vol. 22, no. 2, pp. 105-117, Apr. 1999.

[23] J. G. Kang, D. H. Lee and P. Xirouchakis, "Disassembly sequencing with imprecise data: a case study," Int. J. Ind. Engng.-Theory Appl. Pract., vol, 10, no 2, pp. 407-412, 2003.

[24] Y. Tang and M. C. Zhou, "A Systematic Approach to Design and Operation of Disassembly Lines," IEEE Trans. Automation Sci. Eng., vol. 3, no. 3, pp. 324-329, Jul. 2006.

[25] Y. Tang, "Learning-based disassembly process planner for uncertainty management," IEEE T. Syst. Man. Cy. A., vol. 39, no. 1, pp. 134-143, Jau. 2009.

[26] D. E. Grochowski and Y. Tang, "A Machine Learning Approach for Optimal Disassembly Planning," Int. J. Comput. Integ. M., vol. 22, no. 4, pp. 374-383, 2009.

[27] S. A.Reveliotis, "Uncertainty Management in Optimal Disassembl Planning through Learning-based Strategies," IIE Trans., vol. 30, no. 6, pp. 645-658, 2007.

[28] M. Gao, M. C. Zhou and Y. Tang, "Intelligent Decision Making in Disassembly Process Based on Fuzzy Reasoning Petri Nets," IEEE Trans. Syst., Man, Cybern. B. Cybern., vol. 34, no. 5, pp. 2029-2034, Oct. 2004.

[29] L. Brennan, S. M. Gupta and K. N. Taleb, "Operations planning issues in an assembly/disassembly environment,” Int. J. Oper. Prod. Manag., vol 14, no. 9, pp. 57-67, 1994.

[30] A. J. D. Lambert and S. M. Gupta, Disassembly Modeling for Assembly, Maintenance, Reuse, and Recycling. Boca Raton, Florida: CRC Press, 2005.

[31] M. C. Zhou and F. DiCesare, Petri Net Synthesis for Discrete Event Control of Manufacturing Systems. Norwell, MA: Kluwer, 1993.

[32] M. C. Zhou and K. Venkatesh, Modeling, Simulation and Control of Flexible Manufacturing Systems: A Petri Net Approach. Singpore: World Scientific, 1998.

[33] S. M. McGovern and S. M. Gupta, "The Disassembly Line: Balancing and Modeling", New York: McGraw Hill, 2011.

[34] Y. Tang, M. C. Zhou, E. Zussman and R. J. Caudill, "Disassembly Modeling, Planning and Applications," J. Manuf. Syst., vol. 21, no. 2, pp. 200-217, 2002

[35] S. Bhatnagar, V. K. Mishra and N. Hemachandra, "Stochastic Algorithms for Discrete Parameter Simulation Optimization,” IEEE Trans. Autom. Sci. Eng., vol. 8, no. 4, pp. 780-793, Oct. 2011. 
[36] G. D. Tian, Y. M. Liu, H. W. Zhang, J. W. Chu and G. Xu, "Chance programming models for timeenergy trade-off problem of product disassembly process with multiple stochastic variables," Adv. Sci. Lett., vol. 4, no. 4-5, 2011.

[37] Liu B, Uncertainty Theory: An Introduction to its Axiomatic Foundations. Berlin: Springer-Verlag, 2004.

[38] M. Sedighi and D. Afshari, "Creep feed grinding optimization by an integrated GA-NN system," J. Intell. Manuf., vol. 21, pp. 657-663, 2010.

[39] T. C. Chen and T.T. Sheu, "Model reference robust speed control for induction-motor drive with time delay based on neural network," IEEE Trans. Syst., Man, Cybern., Part A: Syst. Humans, vol. no. 6, pp. 746-753, Nov. 2001

[40] G. D. Tian, J. W. Chu, Y. M. Liu, H. Ke, X. Zhao and G. Xu, "Expected energy analysis for industrial process planning problem with fuzzy time parameters,". Comput. Chem. Eng., vol. 35, no. 12, pp. 2905 2912, 2011

[41] C. A. O. Nascimento, R. Giudici and R. Guardani, "Neural network based approach for optimization of industrial chemical processes," Comput. Chem. Eng.,vol. 24, pp. 2303-2314, 2000.

[42] D. F. Cook, C. T. Ragsdale and R. L. Major, "Combining a neural network with a genetic algorithm for process parameter optimization,” Eng. Appl. Artif. Intell., vol. 13, pp. 391-396, 2000.

[43] G. D. Tian, M. C. Zhou, and J. W. Chu, "A chance constrained programming approach to determine the optimal disassembly Sequence,” IEEE Trans. Autom. Sci. Eng., vol. 10, no. 4, pp. 288-295, Oct. 2013.

[44] G. D. Tian, M. C. Zhou, J. W. Chu, and B. Wang, "Prediction Models of the Number of End-of-life Vehicles in China," in Proc. Int. Conf. Adv. Mech. Sys., Luoyang, China, Sep. 2013, pp. 357-362.

[45] G. D. Tian, T. G. Qiang, J. W. Chu, Guan Xu, and Wei Zhou, "Efficiency optimization for disassembly tools via using NN-GA approach," Math. Probl. Eng., 2013(accepted).

[46] M. A. Ilgin and S. M. Gupta, "Comparison of Economic Benefits of Sensor Embedded Products and Conventional Products in a Multi-product Disassembly Line," Comput. Ind. Eng., vol. 59, pp. 748-763, 2010.

[47] M. A. Ilgin and S. M. Gupta, "Evaluating the impact of sensor-embedded products on the performance of an air conditioner disassembly line," Int. J. Adv. Manuf. Technol., vol. 53, pp. 1199-1216, 2011.

[48] G. D. Tian, J. W. Chu, X. H. Jin, T. Z. Zhang and H.Wang, "Evaluation method and mathematical expression for products disassemblability based on probability theory," (in Chinese) Comput. Integ. M. Syst., vol. 17, no. 6, pp. 1164-1170, 2011.

[49] J. Fischer, Stock. P and G. Zülch, "Simulation of Disassembly and Re-assembly Processes with Betadistributed operation Times," IFIP Int. Fed. Inf. Process, vol. 160, pp. 147-156, 2005.

[50] Y. Tang and M. C. Zhou, Human-in-the-Loop Disassembly Modeling and Planning, Evironmentally Conscious Manufacturing, S.M. Gupta and A. J. D. Lambert, Eds. New York, NY, USA: Taylor \& Fancis, 2008, pp. 363-386.

\section{Authors}

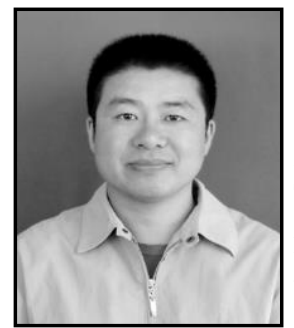

Hongxing Deng received the Ph.D. degrees in physics, automobile application engineering, automobile application engineering respectively, from Northeast Forestry University, Harbin, China, in 2009. He is currently a Professor of Transportation College of Northeast Forestry University. His main research interests include motor vehicle braking, logistics technology.

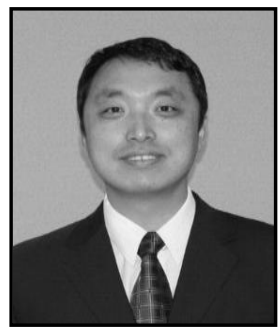

Tiangang Qiang received the B.S., M.S., and Ph.D. degrees in physics, automobile application engineering, automobile application engineering respectively, from Northeast Forestry University, Harbin, China, in 1990, 2003, and 2012, respectively. He is currently a Professor of Transportation College of Northeast Forestry University. His research focuses on vehicle maintenance theory and technology, automotive remanufacturing theory and technology. He has published over 30 journal and conference proceedings papers in the above research areas. 


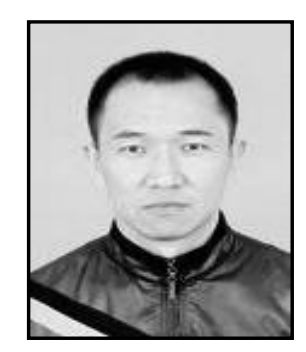

Xiwang Guo received the B.S. degree in computer science and technology from Shenyang Institute of Engineering, Shenyang, China, in 2006, the M.S. degree in Dept. of Aeronautics and Astronautics Manufacturing Engineering. from Shenyang Aerospace University, Shenyang, China, in 2009. He is currently working towards the Ph.D. degree at Northeastern University. He is currently a member of the process industry automation key laboratory of Northeastern University, China and also is a visiting student of Northeast Forestry University, China. His research focuses on remanufacturing, recycling and reuse of automotive, intelligent optimization algorithm. He has published over 10 journal and conference proceedings papers in the above research areas.

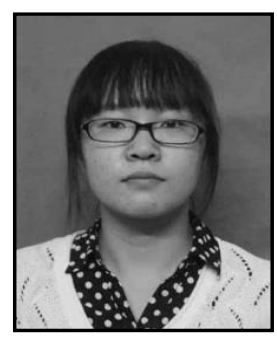

Ying Zhao received the B.S. degree in transportation from Northeast Forestry University, Harbin, China, in 2013. She is currently a graduate of Transportation College, Northeast Forestry University, Harbin, China. Her research focuses on logistics system planning and design, including the positioning of the logistics facilities, distribution route optimization and inventory control 